\title{
Multiextrapolação de Richardson com interpolação aplicada às equações de Navier-Stokes 2D
}

\author{
Márcio A. Martins \\ Departamento de Matemática, Universidade Estadual do Centro Oeste (UNICENTRO-PR) \\ 85040-080, Guarapuava, PR; E-mail: mandre@ unicentro.br
}

Carlos H. Marchi

Departamento de Engenharia Mecânica, Universidade Federal do Paraná (UFPR), Curitiba, PR E-mail: marchi@ufpr.br

\begin{abstract}
Resumo: Analisa-se o desempenho de Multiextrapolação de Richardson (MER) com o objetivo de reduzir e estimar o erro de discretização (Eh) em Dinâmica dos Fluidos Computacional (CFD). Sobre a redução de Eh, propõe-se um conjunto de procedimentos numéricos envolvendo o uso de interpolação polinomial e, em alguns casos, também o emprego de técnicas de otimização. Com relação ao exercício de estimativas para o erro numérico, são analisados dois estimadores que apresentaram resultados acurados, possibilitando assim a determinação de um critério para identificação do desempenho efetivo de MER. Como problema-teste considera-se o trabalho de Shih et al. (1989) sobre o escoamento recirculante de fluido incompressivel com propriedades constantes, em domínio bidimensional, cujo modelo matemático é dado pelas equações de conservação de massa e de Navier-Stokes (2D). O modelo numérico adotado envolve aproximações de primeira e segunda ordens com o método de Volumes Finitos. Os resultados obtidos indicam que a metodologia proposta é promissora inclusive nos casos em que MER é considerada ineficiente na literatura.
\end{abstract}

Palavras-chave: verificação numérica, Multiextrapolação de Richardson, CFD, problema da cavidade, equações de Navier-Stokes.

\section{Introdução}

Atualmente a computação científica desempenha um papel crescente na predição do comportamento de sistemas naturais e artificiais. Muitas vezes, ela é baseada em modelos matemáticos representados por equações diferenciais, como é o caso de CFD. Entretanto, a credibilidade que vem sendo atribuída à CFD é justificada pelo emprego de rigorosos processos de verificação numérica [4].

A verificação numérica tem como objeto de estudo o erro numérico e suas fontes, dentre as quais $E h$ é a mais significativa [12]. As alternativas para reduzir $E h$ são: refinamento de malha, cuja desvantagem é o aumento de memória e tempo computacionais; emprego de métodos de alta ordem, cuja desvantagem é o aumento da complexidade do modelo numérico; e por último, mas não menos importante, a utilização de técnicas de extrapolação das quais a extrapolação de Richardson (ER) é bastante conhecida. Ao se considerar a aplicação da ER de forma recursiva, é possível potencializar a sua eficácia e, denomina-se Multiextrapolação de Richardson (MER) ou em inglês Repeated Richardson Extrapolation (RRE) [7].

Contudo, constam na literatura ([2], [6] e [10]) relatos sobre dificuldades inerentes ao emprego de MER em algumas variáveis de interesse. Nesse sentido, o texto [8] traz contribuições significativas. Outro desafio a ser considerado diz respeito ao emprego de MER em situações realísticas, ou seja, em modelos matemáticos cujas soluções analíticas são desconhecidas. Nessa perspectiva, o emprego de estimadores para o erro numérico é recomendado. Com essa finalidade, em [9] é realizado um estudo envolvendo a resolução numérica das equações de Poisson, Advecção-difusão e Burgers (2D), no qual são indicadas alternativas.

O presente trabalho consiste em uma extensão a essas propostas ([8] e [9]), ou seja, no emprego de metodologia para redução e estimativa de $E h$ inerente ao cálculo de diversas variáveis 
de interesse, incluindo casos em que MER era considerada ineficiente. Nesse encalço, considerase o texto [14] (problema da cavidade quadrada com tampa móvel), cuja utilização é frequente na obtenção de resultados numéricos de referência (benchmarks). Como problema-teste adota-se, então, o emprego do método de Volumes Finitos na resolução das equações de conservação de massa e de Navier-Stokes (2D), de acordo com a descrição apresentada na sequência.

\section{Problema-teste}

\section{Modelo matemático}

O modelo matemático adotado envolve as leis de conservação de massa e quantidade de movimento linear (equações de Navier-Stokes) no contexto do problema clássico do escoamento recirculante em uma cavidade quadrada com tampa móvel. As simplificações consideradas são: estado permanente, escoamento laminar bidimensional nas direções $x$ e $y$, fluido incompressível, $\rho$ (densidade) e $\mu$ (viscosidade) constantes, e sendo desconsiderados outros efeitos. Assim,

$$
\begin{aligned}
& \frac{\partial u}{\partial x}+\frac{\partial v}{\partial y}=0 \\
& \rho \frac{\partial\left(u^{2}\right)}{\partial x}+\rho \frac{\partial(u v)}{\partial y}=\mu\left(\frac{\partial^{2} u}{\partial x^{2}}+\frac{\partial^{2} u}{\partial y^{2}}\right)-\frac{\partial p}{\partial x} \\
& \rho \frac{\partial(u v)}{\partial x}+\rho \frac{\partial(v)^{2}}{\partial y}=\mu\left(\frac{\partial^{2} v}{\partial x^{2}}+\frac{\partial^{2} v}{\partial y^{2}}\right)-\frac{\partial p}{\partial y}+S,(x, y) \in[0,1]
\end{aligned}
$$

em que $p$ representa a pressão e $S$ o termo fonte dado em [14] no problema com solução analítica fabricada, com condições de contorno do tipo Dirichlet. As variáveis de interesse para esse problema envolvem as variáveis primitivas $u$ e $v$, bem como a integral dessas. Mais especificamente, considera-se: $u c=u(1 / 2,1 / 2) ; \psi \min =$ valor mínimo da função de corrente para $(x, y) \in[0,1]$ e sua ordenada $y ; u$ min $=$ valor mínimo do perfil de $u$ em $x=1 / 2$ e sua respectiva coordenada $y ; v \max =$ valor máximo do perfil de $v$ em $y=1 / 2$ e sua respectiva coordenada $x$.

\section{Modelo numérico}

A resolução numérica do modelo matemático descrito pelas Eqs. (1) a (3) é obtida com os seguintes parâmetros: (1) método de Volumes Finitos [3]; (2) aproximação por diferença central (CDS) [3] para os termos difusivos e de pressão; (3) aproximação CDS com correção adiada [3] sobre a aproximação com diferença atrasada (UDS) para os termos advectivos; (4) a partir disso, as Eqs. (1) a (3) são resolvidas com os métodos MSI (Modified Strongly Implicit) [13] e SIMPLEC (Semi IMPlicit Linked Equations Consistent) [15] para o tratamento do acoplamento pressão-velocidade; (5) malha uniforme; (6) condições de contorno implementadas com a técnica de volumes fictícios [3]; (7) as Eqs. (1) a (3) são escritas em regime transiente, visando o uso do tempo somente como um parâmetro de relaxação no processo de solução iterativa do modelo matemático discretizado. Esse modelo numérico não requer o emprego de condições de contorno para a pressão [6] e as expressões para os nós internos são estendidas para os contornos.

\section{Metodologia}

O erro numérico $(E)$ pode ser definido como a diferença entre a solução analítica $(\Phi)$ de uma variável de interesse e a sua solução numérica $(\phi)$. Entretanto ao se considerar o emprego de um método de discretização em um domínio de cálculo $\Omega$, Eh pode ser considerado a principal fonte de $E$ [11], e com essa perspectiva segue a sua representação [7] 


$$
E=\Phi-\phi \cong E h=k_{0} h^{p_{0}}+k_{1} h^{p_{1}}+k_{2} h^{p_{2}}+k_{3} h^{p_{3}}+\ldots,
$$

em que os coeficientes $k_{j}, j=0,1,2,3, \ldots$ são números reais obtidos em função da variável dependente (do problema) e de suas derivadas, mas independem de $h$ (espaçamento entre os pontos nodais da malha $\Omega^{h}$ ).

Considerando-se a obtenção de solução numérica para determinada variável de interesse $(\phi)$, em $G$ malhas distintas $\Omega^{h_{1}}, \Omega^{h_{2}}, \ldots, \Omega^{h_{g}}, \ldots, \Omega^{h_{G}}$, geradas com razão de refino $r=h_{g-1} / h_{g} \quad(g=2, \ldots, G)$, o emprego de MER com $m$ níveis de extrapolação é dado por [8]:

$$
\phi_{g, m}=\phi_{g, m-1}+\frac{\phi_{g, m-1}-\phi_{g-1, m-1}}{r^{p_{m-1}-1}} ; g=2, \ldots, G ; m=1, \ldots, g-1
$$

No presente estudo são considerados diferentes tipos de $\phi$, de acordo com a sua localização em malhas distintas, conforme detalhado em [8]. Com esse enfoque, e seguindo a definição apresentada na Eq. (4) considera-se

$$
E m=\Phi-\phi_{g, m},
$$

ou seja, $E m$ representa o $E$ resultante da metodologia investigada neste trabalho.

Com relação às estimativas para $E h$ e $E m$, são adotados, respectivamente, os estimadores multicoeficiente $\left(U_{m c}\right)$ e psi corrigido $\left(U_{\psi^{*}}\right)$ [9]. A concepção de $U_{m c}$ admite que a estimativa para $E h$ é composta por $m=g-1$ termos, com base na Eq. (4). Propõe, então: para $g=2$ $E h\left(\phi_{2}\right) \approx \phi_{2,1}-\phi_{2}=k_{0} h_{2}{ }^{p} \quad$ (estimativa monocoeficiente); para $g=3 \quad E h\left(\phi_{3}\right) \approx \phi_{3,2}-\phi_{3}$ $=k_{0} h_{3}{ }^{p_{0}}+k_{1} h_{3}{ }^{p_{1}}$ (estimativa bicoeficiente); e assim por diante,

$$
U_{m c}\left(\phi_{g}\right)=\phi_{g, m}-\phi_{g} ; g=2, \ldots, G ; m=g-1 \text {. }
$$

Considerando-se a série de Richardson $\left(R_{\infty}\right)$ [5], em que $\psi \in \Re$ corresponde à razão de convergência, em [9] propõe-se

$$
U_{\psi^{*}}\left(\phi_{g, m}\right)=\frac{\phi_{g, m}-\phi_{g-1, m-1}}{\psi^{*}-1}, g=2, \ldots, G
$$

sendo que o seu cálculo envolve a determinação do parâmetro $\psi^{*}$ (Eq. (9)) que,

$$
\psi^{*}=\left\{\begin{array}{l}
\frac{\phi_{g, m}-\phi_{g-1, m-1}}{\phi_{g+1, m+1}-\phi_{g, m}}, g=2,3, \ldots G-1 ; \\
\frac{\left(\phi_{g-1, m-1}-\phi_{g-2, m-2}\right)^{2}}{\left(\phi_{g, m}-\phi_{g-1, m-1}\right)\left(\phi_{g-2, m-2}-\phi_{g-3, m-3}\right)}, g=G .
\end{array}\right.
$$

por sua vez, indica se MER é eficaz na redução de $E$, isto é, deve satisfazer a condição $\left|\psi^{*}\right|>1$.

\section{Resultados e Conclusão}


Analisou-se a metodologia proposta sobre diversas variáveis, conforme descrito na seção (2). As Figuras 1 (a) e 1 (b) representam, respectivamente, os resultados obtidos, em variável que possui localização fixa e nodal $(u c)$ em todas as malhas adotadas, e variável que possui alteração de coordenada em malhas distintas ( $\psi$ min) (ponto extremo). No segundo caso, para determinação de $\phi_{g, m}$ (com MER) considera-se, também, o emprego de interpolação polinomial (2D) de sexto grau e do método do Gradiente Conjugado com a busca linear de Armijo [1].

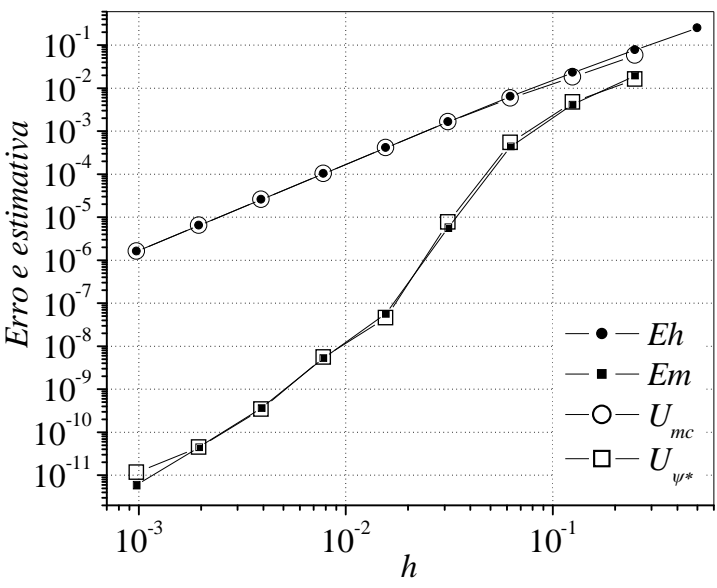

(a) $\phi$ com localização nodal (fixa), $u c$

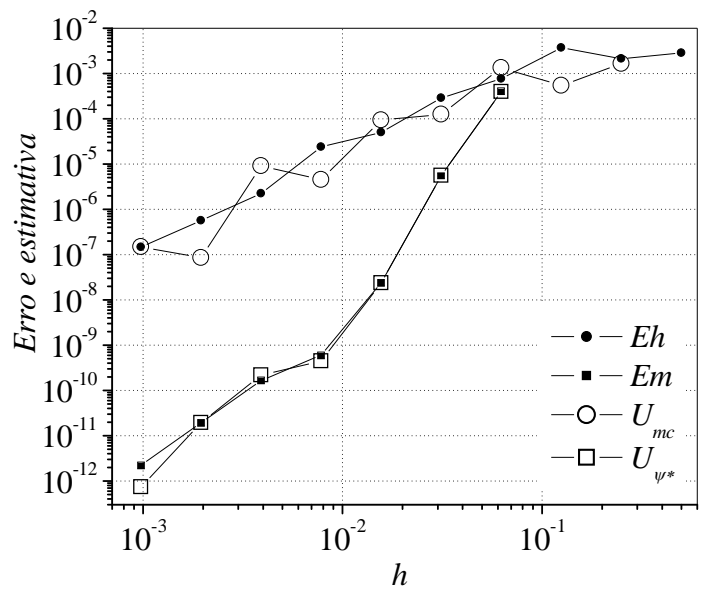

(b) $\phi$ com localização não nodal (móvel), $\psi$ min

Figura 1: Eh (sem MER), Em (com MER) e suas respectivas estimativas.

Observa-se na Figura 1 (a) que: Em possui magnitude significativamente menor do que $E h$; os estimadores $U_{m c}$ e $U_{\psi^{*}}$ mostraram-se acurados, uma vez que apresentam correlação direta com os resultados obtidos para $E h$ e $E m$, respectivamente.

Na Figura 1 (b), nota-se primeiramente que $E h$ não possui um comportamento assintótico (desejável). Tal comportamento está relacionado com a mudança de coordenada de $\phi$ em malhas distintas, ou seja, com o processo de refinamento de malha a obtenção de $\phi=\psi \min$ (valor extremo) acarreta em localização distinta. Com isso, $U_{m c}$ torna-se inacurado porém sua ordem de magnitude é compatível com a de Eh. Essa situação é mencionada na literatura como um caso de anomalia em que MER não é capaz de reduzir $E h$. No entanto, ao se empregar interpolação polinomial sobre os dados nodais obtidos e a partir da função polinomial resultante determinar o seu ponto extremo ( $\phi=\psi$ min ), o comportamento assintótico de Eh é alcançado. Dessa forma, MER pode ser empregada e resulta em um comportamento semelhante ao descrito anteriormente. Além disso, $U_{\psi^{*}}$ retrata com uma acurácia expressiva o comportamento do Em atingido.

Para as demais variáveis de interesse é possível observar na Tabela 1 os resultados obtidos para $E h$ e $E m$, assim como para $U_{m c}$ e $U_{\psi^{*}}$, na malha com espaçamento $h=1,9531 \times$ $10^{-3}$ entre os pontos nodais.

Tais resultados ilustram a eficácia da metodologia estudada, no presente trabalho, no sentido em que: (1) o modelo matemático analisado é representativo no âmbito de CFD por contemplar aspectos de não linearidade e de acoplamento de variáveis; (2) alcançou-se uma redução expressiva do erro numérico, com o emprego de MER, mesmo em situações desencorajadas na literatura; (3) as propostas de estimativas para o erro numérico apresentaram acurácia significativa e ilustram alternativas a serem utilizadas em situações realísticas, ou seja, em modelos matemáticos cuja solução analítica não seja conhecida.

\begin{tabular}{lllll}
\hline Variável & $E h$ & $E m$ & $U_{m c}$ & $U_{\psi^{*}}$ \\
\hline
\end{tabular}




\begin{tabular}{ccccc}
\hline$u c$ & $-6,457257 \mathrm{E}-06$ & $4,491869 \mathrm{E}-11$ & $-6,457302 \mathrm{E}-06$ & $4,441644 \mathrm{E}-11$ \\
$\psi$ min & $-5,682980 \mathrm{E}-07$ & $-1,940962 \mathrm{E}-11$ & $-8,478479 \mathrm{E}-08$ & $-1,944004 \mathrm{E}-11$ \\
$y(\psi \min )$ & $7,553119 \mathrm{E}-05$ & $3,425862 \mathrm{E}-12$ & $1,280862 \mathrm{E}-04$ & $3,539677 \mathrm{E}-12$ \\
$u$ min & $-3,821675 \mathrm{E}-06$ & $1,447587 \mathrm{E}-11$ & $-4,428316 \mathrm{E}-07$ & $1,433716 \mathrm{E}-11$ \\
$y(u \min )$ & $-9,313970 \mathrm{E}-04$ & $-1,124376 \mathrm{E}-10$ & $5,018021 \mathrm{E}-04$ & $-1,115581 \mathrm{E}-10$ \\
$v \max$ & $4,925976 \mathrm{E}-06$ & $1,159134 \mathrm{E}-10$ & $6,401623 \mathrm{E}-06$ & $1,158047 \mathrm{E}-10$ \\
$x(v \max )$ & $-5,891971 \mathrm{E}-04$ & $-5,580941 \mathrm{E}-11$ & $-3,777327 \mathrm{E}-04$ & $1,158047 \mathrm{E}-10$ \\
\hline
\end{tabular}

Tabela 1: Resultados para o erro numérico obtido em malha $512 \times 512$, com e sem MER, bem como suas respectivas estimativas.

\section{Agradecimentos}

Os autores agradecem o apoio financeiro do CNPq (Conselho Nacional de Desenvolvimento Científico e Tecnológico), AEB (Agência Espacial Brasileira) através do Programa Uniespaço, Fundação Araucária (Paraná) e CAPES (Coordenação de Aperfeiçoamento de Pessoal de Nível Superior). O primeiro autor agradece à Universidade Estadual do Centro-Oeste (UNICENTRO). O segundo autor é bolsista do CNPq.

\section{Referências}

[1] M. S. Bazaraa, H. D. Sherali, C. M. Shetty, "Nonlinear programming: theory and algorithms", Wiley \& Sons, New York, 2006.

[2] C. Burg, T. Erwin, Application of Richardson extrapolation to the numerical solution of partial differencial equations, Num. Meth. Part. Diff. Eq., vol. 25, pp. 810-832, (2009).

[3] J. H. Ferziger, M. Peric, "Computational methods for fluid dynamics", Springer, New York, 2002.

[4] M. Karimi, G. Akdogan, K. H. Dellimore, S. M. Bradshaw, Quantification of Numerical Uncertainty in Computational Fluid Dynamics Modelling of Hydrocyclones. Comp. and Chem. Eng., vol. 42, pp. 3671-3694, (2012).

[5] C. H. Marchi, A. F. C. Silva, Multidimensional discretization error estimation for convergent apparent order. J. Braz. Soc. Mech. Sc. Eng., vol. 27, pp. 432-439, (2005).

[6] C. H. Marchi, R. Suero, L. K. Araki, The lid-driven square cavity flow: numerical solution with a 1024 x 1024 grid, J. Braz. Soc. of Mech. Sc. and Eng., vol. 31, pp. 186-198, (2009).

[7] C. H. Marchi, L. K. Araki, L. A. Novak, C. D. Santiago, A. P. S. Vargas, Highly accurate numerical solutions with repeated Richardson extrapolation for 2D Laplace equation, Appl. Math. Mod., vol. 37, pp. 7386-7397, (2013).

[8] M. A. Martins, C. H. Marchi, L. A. Novak, M. A. V. Pinto, L. K. Araki, S. F. T. Gonçalves, Multiextrapolação de Richardson com interpolação para reduzir o erro de discretização em CFD, I CMAC-SE, Bauru, Brasil, 2013.

[9] M. A. Martins, C. H. Marchi, M, A. V. Pinto, L. K. Araki, Estimativa para o erro de discretização com o emprego de Multiextrapolação de Richardson em CFD, I CMAC-Sul, Curitiba, Brasil, 2014.

[10] X. Nicolas, M. Medale, S. Glockner, S. Gounand, Benchmark solution for a threedimensional mixed-convection flow, part 1: reference solutions, Num. Heat Transf., Part B, vol. 60, pp. 325-345, (2011).

[11] J. C. Roy, Review of code and solution verification procedures for computational simulation, J. Comp. Phys, vol. 205, pp. 131-156, (2005).

[12] J. C. Roy, W. L. Oberkampf, A comprehensive framework for verification, validation, and uncertainty quantification in scientific computing, Comp. Meth. in Appl. Mech. Eng., vol. 200, pp. 2131-2144, (2011).

[13] G. E. Schneider, M. Zedan, A modified strongly implicit procedure for the numerical solution of field problems, Nume. Heat Transf., vol. 4, pp. 1-19, (1981). 
[14] T. M. Shih, C. H. Tan, B. C. Hwang, Effects of grid staggering on numerical scheme, Int. J. Num. Meth. Fld., vol. 9, pp. 193-212, (1989).

[15] J. P. Van Doormaal, G. D. Raithby, Enhancements of the SIMPLE method for predicting incompressible fluid flow, Num. Heat Transf., vol. 7, pp. 147-163 (1984). 